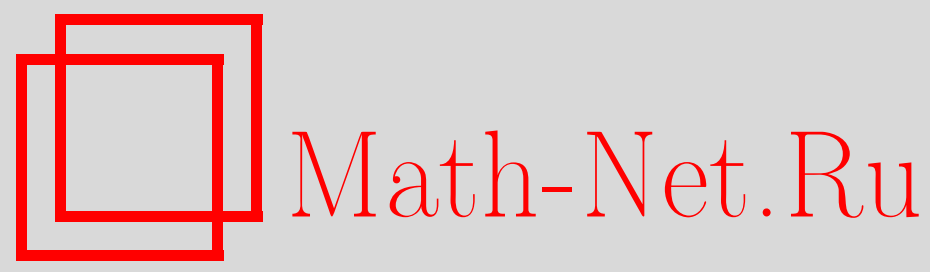

Г. М. Айрапетян, О разрешимости задачи Дирихле с граничными функциями из пространств с весом, Матем. заметки, 2004, том 76, выпуск 5, 643-650

DOI: https://doi.org/10.4213/mzm135

Использование Общероссийского математического портала Math-Net.Ru подразумевает, что вы прочитали и согласны с пользовательским соглашением http://www . mathnet.ru/rus/agreement

Параметры загрузки:

IP : 54.157 .27 .8

26 апреля 2023 г., $15: 53: 21$

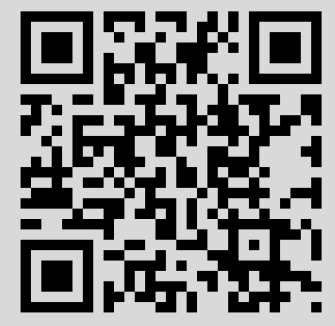




\section{О РАЗРЕШИМОСТИ ЗАДАЧИ ДИРИХЛЕ С ГРАНИЧНЫМИ ФУНКЦИЯМИ ИЗ ПРОСТРАНСТВ С ВЕСОМ}

\section{Г.М. Айрапетян}

Исследуется задача Дирихле в полуплоскости в весовых пространствах, когда весовая функция имеет единственную особенность в бесконечно удаленной точке.

Библиография: 13 названий.

1. Пусть $B_{1}-$ класс гармонических функций $u(z)$ в верхней полуплоскости $G^{+}=\{z$ : $\operatorname{Im} z>0\}$, удовлетворяющих для любого $y_{0}>0$ неравенству

$$
|u(z)|<C \exp |z|^{\gamma}, \quad \gamma<1, \quad \operatorname{Im} z>y_{0}>0,
$$

где $C$ - постоянная, не зависящая от $x=\operatorname{Re} z$, но зависящая, вообще говоря, от $y_{0}$. B работе рассматривается задача Дирихле в классе $B_{1}$ в следующей постановке: определить действительную гармоническую функцию $u(x, y) \in B_{1}$ так, чтобы имело место граничное условие

$$
\lim _{y \rightarrow+0}\|u(x, y)-f(x)\|_{L^{1}(\rho)}=0
$$

где $\rho(x)$ - измеримая неотрицательная функция на действительной оси такая, что $\rho(x) \in$ $L^{\infty}(-A, A), \rho^{-1}(x) \in L^{\infty}(-A, A)$ для любого $A>0$.

Число

$$
\alpha=\sup \left\{\beta: \rho(x)(1+|x|)^{\beta} \in L^{\infty}(-\infty,+\infty)\right\}<\infty
$$

будем назьвать порядком особенности $\rho(x)$ в бесконечно удаленной точке. Всюду в дальнейшем будем предполагать, что $0 \leqslant \alpha<\infty$.

Граничные задачи в классах аналитических функций и тесно связанная с ними теория сингулярных интегралов в весовых пространствах $L^{p}(d \mu)\left(d \mu=\rho(x) d x+d \mu_{s}\right.$, где $d \mu_{s}$ - сингулярная часть) исследованы в многочисленных работах (см. [1]-[8]). Было установлено, что для ограниченности сингулярного оператора в пространствах $L^{p}(d \mu)$, $p>1$, необходимо и достаточно, чтобы мера $d \mu$ была абсолютно непрерьвной и $\rho(x)$ удовлетворяла условию Макенхаупта (см. [6]). Отметим, что если мера удовлетворяет этому условию, то функция $f$ из класса $L^{p}(d \mu)$ является также абсолютно интегрируемой по мере Лебега, т.е. принадлежит классу $L^{1}$. Однако граничные задачи в классах аналитических функций и, в частности, задача Дирихле, когда граничные условия понимаются в смысле средней сходимости $L^{p}(d \mu)($ cм. [9]-[12]), допускают такое жеполное исследование в весовьх пространствах при нарушении условия Макенхаупта. Задача Дирихле в единичном круге, когда весовая функция имеет конечное число особенностей 
конечного порядка, исследована в [9]. Там же описаны весовые функции, обеспечивающие разрешимость этой задачи для любой функции $f \in L^{p}(\rho(x) d x)$. Задача Дирихле для $R O$-меняющейся (определение см. ниже) в особьх точках весовой функции исследована в [10]. Задача (2) в полуплоскости в случае степенной весовой функции (вида $O\left(\left|x-x_{0}\right|^{\alpha}\right)$, где $\alpha$ - неотрицательное целое число) была исследована в [11].

В настоящей работе задача (2) исследуется в предположении, что $\rho(x) R O$-меняющаяся в бесконечно удаленной точке.

Функцию $g(x)$, определенную в $\left(A_{0}, \infty\right)$, будем назьвать $R O$-меняющейся в бесконечно удаленной точке слева [13], если ее можно представить в виде

$$
g(x)=\exp \left(g_{1}(x)+\int_{A_{1}}^{x} \frac{g_{2}(t)}{t} d t\right), \quad x \in\left(A_{0}, \infty\right)
$$

где $A_{1}>A_{0}$, а $g_{1}(x)$ и $g_{2}(x)$ - измеримые и ограниченные функции на $\left(A_{0}, \infty\right)$. Аналогично определяется класс функций $R O$-меняющихся в бесконечно удаленной точке справа. Если функция в бесконечно удаленной точке является $R O$-меняющейся слева и $R O$-меняющейся справа, то скажем, что функция $R O$-меняющаяся в бесконечно удаленной точке. Функцию $\rho(x)$ отнесем к классу $R$, если функция

$$
\rho_{1}(x)=(1+|x|)^{\alpha} \rho(x)
$$

$R O$-меняющаяся в бесконечно удаленной точке, причем функция $g_{2}(x)$ из представления (4) удовлетворяет соотношениям

$$
\varlimsup_{|x| \rightarrow \infty} g_{2}(x)<1-\{\alpha\}, \quad \lim _{|x| \rightarrow \infty} g_{2}(x)>-\{\alpha\}
$$

если $\alpha$ - нецелое число, и

$$
\varlimsup_{|x| \rightarrow \infty} g_{2}(x) \leqslant 0, \quad \varliminf_{|x| \rightarrow \infty} g_{2}(x)>-1
$$

если $\alpha$ - целое число.

2. ЛЕмма 1. Пусть $k>0$ - иелое число. Тогда

$$
\left|(x+i y)^{k}-(x-i y)^{k}\right|<C y\left(y^{k-1}+|x|^{k-1}\right) .
$$

Ecлu $|x|>2 k y$, mo

$$
\left|(x+i y)^{k}-(x-i y)^{k}\right|>C_{1} y|x|^{k-1},
$$

әде $C, C_{1}>0$ - некоторье постоянные. 
ДокАЗАТЕЛЬСТво. Пусть $|x|>y$. Тогда $|x+i y|<2|x|,|x-i y|<2|x|$ и

$$
\left|(x+i y)^{k}-(x-i y)^{k}\right|<2 y \sum_{j=0}^{k-1}|x+i y|^{j}|x-i y|^{k-j-1}<C y|x|^{k-1} .
$$

Если $|x|<y$, то $|x+i y|<2|y|,|x-i y|<2|y|$ и $\left|(x+i y)^{k}-(x-i y)^{k}\right|<C y^{k}$, и (8) доказано. Предположим $|x|>2 k y$; тогда

$$
\begin{aligned}
\left|(x+i y)^{k}-(x-i y)^{k}\right| & =|x+i y|^{k}\left|1-\left(\frac{x-i y}{x+i y}\right)^{k}\right| \\
& =y|x+i y|^{k-1} \sum_{j=0}^{k-1}\left|\frac{x-i y}{x+i y}\right|^{j}(\cos j \varphi+i \sin j \varphi),
\end{aligned}
$$

где $\varphi=2 \arg (x-i y)$. Из условия $|x|>2 k y$ следует, что $\cos j \varphi>0, j=0,1, \ldots, k-1$; следовательно, (9) доказано.

Функцию $g(x)$ на промежутке $(a, b)$ будем называть почти монотонно возрастающей, если существует $A>0$ такое, что для любых $x^{\prime}<x^{\prime \prime}$ из этого промежутка $g\left(x^{\prime}\right)<A g\left(x^{\prime \prime}\right)$. Аналогично определяется почти монотонно убывающая функиия. В следующей лемме собраны свойства функции $\rho(x) \in R$.

Лемма 2. Пусть $\rho(x) \in R$. Справедливы следующие утверждения.

а) Пусть $\alpha$ - нецелое число. Тогда существуют $\delta_{0} \in\left(0,1-\left\{\alpha_{0}\right\}\right), \delta_{1} \in\left(-\left\{\alpha_{0}\right\}, 0\right)$ такие, что для любого $\delta>\delta_{0}$ и для любого числа $A$ функиия $|x+i|^{\delta} \rho_{1}(x)$ почти монотонно возрастает в промежутке $(A, \infty)$ и почти монотонно уббввает $в$ промежутке $(-\infty, A)$. Соответственно $|x+i|^{-\delta} \rho_{1}(x)$ почти монотонно убывает на $(A, \infty)$ и почти монотонно возрастает на $(-\infty, A)$ для любого $\delta<\delta_{1}$.

b) Если $\alpha$ - иелое число, то для любого числа $A$ функция $|x+i| \rho_{1}(x)$ почти монотонно возрастает на $(A, \infty)$ и почти монотонно убъвает на $(-\infty, A) ;|x+i|^{\delta} \rho_{1}(x)$ почти монотонно убыв вает на $(A, \infty)$ и почти монотонно возрастает на $(-\infty, A)$ для любого $\delta<0$.

c) Для любого $\delta>0$

$$
\int_{-\infty}^{\infty} \frac{\rho_{1}(x) d x}{(1+|x|)^{1+\delta}}<\infty
$$

ДокАЗАТЕЛЬСТво. Пусть согласно $(6) \delta_{0}$ выбрано так, чтобы имело место

$$
\varlimsup_{|x| \rightarrow \infty} g_{2}(x)<\delta_{0}<1-\left\{\alpha_{k}\right\} .
$$

Тогда если $A<x^{\prime}<x^{\prime \prime}$, то при $\delta>\delta_{0}$ из (4) имеем

$$
\begin{aligned}
\frac{\left|x^{\prime}+i\right|^{\delta} \rho_{1}\left(x^{\prime}\right)}{\left|x^{\prime \prime}+i\right|^{\delta} \rho_{1}\left(x^{\prime \prime}\right)} & =e^{g\left(x^{\prime}\right)-g\left(x^{\prime \prime}\right)} \exp \left(\int_{x^{\prime}}^{x^{\prime \prime}} \frac{\delta-g_{2}(t)}{t} d t\right) \\
& <A \exp \left(\int_{x^{\prime}}^{x^{\prime \prime}} \frac{\delta-g_{2}(t)}{t} d t\right) .
\end{aligned}
$$

Поэтому $\left|x^{\prime}+i\right|^{\delta} \rho_{1}\left(x^{\prime}\right)\left(\left|x^{\prime \prime}+i\right|^{\delta} \rho_{1}\left(x^{\prime \prime}\right)^{-1}<C\right.$.

Аналогично доказьваются остальные утверждения a), b) леммы. Утверждение c) непосредственно следует из определения класса $R$. 
ЛЕМма 3. Пусть

$$
g(x)=\exp \left(\int_{A}^{x} \frac{g_{2}(t) d t}{t}\right),
$$

где $g_{2}(x)$ - ограниченная функиия на $(A, \infty)$. Тогда если $y \in\left(2^{-1} x, 2 x\right)$, то

$$
\left|\frac{g(x)-g(y)}{x-y}\right|<C\left|\frac{g(x)}{x}\right| \text {. }
$$

ДоКАЗАТЕЛЬСТВо. Из определения функции $g(x)$ имеем

$$
\left|\frac{g(x)-g(y)}{x-y}\right|=\left|\frac{g(x)}{x-y}\right|\left(1-\exp \left(\int_{x}^{y} \frac{g_{2}(t) d t}{t}\right)\right) .
$$

Так как $2^{-1}<y x^{-1}<2$, то

$$
\int_{x}^{y} \frac{g_{2}(t) d t}{t}<A \ln 2
$$

Поэтому

$$
\begin{aligned}
\left|\frac{g(x)-g(y)}{x-y}\right| & <C\left|\frac{g(x)}{x-y}\right|\left|\int_{x}^{y} \frac{g_{2}(t) d t}{t}\right| \\
& <C\left|\frac{g(x)}{x-y}\right|\left|\ln \frac{y}{x}\right|=C\left|\frac{g(x)}{x-y}\right|\left|\ln \left(1-\frac{y-x}{x}\right)\right| .
\end{aligned}
$$

Учитьвая, что $|y-x||x|^{-1}<1$, завершаем доказательство леммы.

Лемма 4. Пусть $\rho(x) \in R$. Тогда

$$
\begin{aligned}
& \sup _{t \in(-\infty, \infty)} \frac{|t+i|^{\left\{\alpha_{0}\right\}}}{\rho_{1}(t)} \int_{-\infty}^{\infty} \frac{y \rho_{1}(x) d x}{\left((x-t)^{2}+y^{2}\right)|x+i|^{\left\{\alpha_{0}\right\}}}<\infty \\
& \sup _{t \in(-\infty, \infty)} \frac{|t+i|^{\left\{\alpha_{0}\right\}}}{\rho_{1}(t)} \int_{-\infty}^{\infty} \frac{y \rho_{1}(x) d x}{|t-x-i y||x+i|^{1+\left\{\alpha_{0}\right\}}}<\infty .
\end{aligned}
$$

ДокАЗАтЕЛЬСтво. Пусть $t \in(0, \infty)$. В силу леммы 2 существует $\delta \in\left(0,1-\left\{\alpha_{0}\right\}\right)$ такое, что функция $|x+i|^{\delta} \rho_{1}(x)$ почти монотонно возрастает на $(0, \infty)$. Поэтому

$$
\begin{aligned}
& \frac{|t+i|^{\left\{\alpha_{0}\right\}}}{\rho_{1}(t)} \int_{-\infty}^{2^{-1} t} \frac{y \rho_{1}(x) d x}{\left((x-t)^{2}+y^{2}\right)|x+i|^{\left\{\alpha_{0}\right\}}} \\
& \quad<C\left(1+\frac{|t+i|^{\left\{\alpha_{0}\right\}}}{\rho_{1}(t)} \int_{0}^{2^{-1} t} \frac{y|x+i|^{\delta} \rho_{1}(x) d x}{|t+i|^{2}|x+i|^{\delta+\left\{\alpha_{0}\right\}}}\right)<C_{1} .
\end{aligned}
$$

Так как $\rho(x) \in R$, то $\rho_{1}(x)$ представима в виде (4). Не нарушая общности, можно предположить, что $g_{1}(x) \equiv 0$. Применяя лемму 3 , получим

$$
\begin{aligned}
& \frac{|t+i|^{\left\{\alpha_{0}\right\}}}{\rho_{1}(t)} \int_{2^{-1} t}^{2 t} \frac{y \rho_{1}(x) d x}{\left((x-t)^{2}+y^{2}\right)|x+i|^{\left\{\alpha_{0}\right\}}} \\
& \quad=\left|\frac{|t+i|^{\left\{\alpha_{0}\right\}}}{\rho_{1}(t)} \int_{2^{-1} t}^{2 t} \frac{y\left(\rho_{1}(x)-\rho_{1}(t)\right) d x}{\left((x-t)^{2}+y^{2}\right)|x+i|^{\left\{\alpha_{0}\right\}}}+\int_{2^{-1} t}^{2 t} \frac{y|t+i|^{\left\{\alpha_{0}\right\}} d x}{\left((x-t)^{2}+y^{2}\right)|x+i|^{\left\{\alpha_{0}\right\}} \mid}\right| \\
& \quad<A\left(1+\int_{2^{-1} t}^{2 t} \frac{y|t+i|^{\left\{\alpha_{0}\right\}}|x-t| d x}{\left((x-t)^{2}+y^{2}\right)|x+i|^{\left\{\alpha_{0}\right\}}}\right)<A_{1}\left(1+\frac{y}{t} \ln \frac{t^{2}+y^{2}}{y^{2}}\right)<A_{2} .
\end{aligned}
$$


В силу леммы $2 \delta \in(-\{\alpha\}, 0)$ можем подобрать так, чтобы функция $|x+i|^{\delta} \rho_{1}(x)$ была почти монотонно убьвающей на $(0, \infty)$, и поэтому

$$
\frac{|t+i|^{\left\{\alpha_{0}\right\}}}{\rho_{1}(t)} \int_{2 t}^{\infty} \frac{y \rho_{1}(x) d x}{\left((x-t)^{2}+y^{2}\right)|x+i|^{\left\{\alpha_{0}\right\}}}<C \int_{2 t}^{\infty} \frac{y|t+i|^{\{\alpha\}+\delta} d x}{\left((x-t)^{2}+y^{2}\right)|x+i|^{\left\{\alpha_{0}\right\}+\delta}}<C_{1}
$$

Утверждение $(11)$ леммы при $t \in(0, \infty)$ доказано. Аналогично устанавливаются другие утверждения.

3. Пусть $\rho^{\prime}(x)=(1+|x|)^{-n_{0}}, x \in(-\infty, \infty)$, где $n_{0}=[\alpha]+1$. Если $u(x, y)$ удовлетворяет условию (2), то из леммы 2 следует, что

$$
\lim _{y \rightarrow+0}\|u(x, y)-f(x)\|_{L^{1}\left(\rho^{\prime}\right)}=0
$$

поэтому $u(x, y)$ можно представить в виде (см. [12])

$$
u(x, y)=u_{0}(x, y)+u_{1}(x, y)
$$

где

$$
\begin{aligned}
u_{0}(x, y)= & \operatorname{Re} \sum_{p=0}^{n_{0}} i a_{p} z^{p} \\
u_{1}(x, y)= & \operatorname{Re}\left(\frac{(z+i)^{n_{0}-1}}{4 \pi i} \int_{-\infty}^{\infty} \frac{f(t) d t}{(t+i)^{n_{0}-1}(t-z)}\right) \\
& +\operatorname{Re}\left(\frac{(z-i)^{n_{0}-1}}{4 \pi i} \int_{-\infty}^{\infty} \frac{f(t) d t}{(t-i)^{n_{0}-1}(t-z)}\right)
\end{aligned}
$$

причем $a_{p}, p=0,1, \ldots, n_{0},-$ произвольные действительные числа.

Скажем, что функция $\rho(x)$ удовлетворяет условию $R_{0}$, если $\alpha$ - нецелое число, либо $\alpha$ - целое число и вьполняется соотношение

$$
\int_{-\infty}^{\infty} \frac{\rho_{1}(x) d x}{1+|x|}<\infty
$$

Теорема 1. Пусть $\rho(x) \in R$. Тогда справедливы следующие утвержсдения.

а) Если $\rho(x)$ удовлетворяет условию $R_{0}$, то общее решение однородной задачи (2) можно представить в виде (15).

b) Пусть $\rho(x)$ не удовлетворяет условию $R_{0}$. Тогда общее решение однородной задачи (2) мохсно представить в виде (15) при $a_{n_{0}}=0$.

ДоКАЗАТЕЛЬСТво. Пусть $u(x, y)$ - произвольное решение однородной задачи $(2)$. Так как $\rho^{\prime}(x)(\rho(x))^{-1} \in L^{\infty}(-\infty, \infty)$, из $(2)$ при $f \equiv 0$ имеем

$$
\lim _{y \rightarrow+0}\|u(x, y)\|_{L^{1}\left(\rho^{\prime}\right)}=0
$$

поэтому $u(x, y)$ представима в виде $(15)$, где $a_{p}$ - некоторые действительные числа. Для доказательства утверждения а) теоремы нам предстоит доказать, что функция (15) 
удовлетворяет однородному условию (2) для любого действительного числа $a_{p}$. Сначала предположим, что число $\alpha$ нецелое. Достаточно рассмотреть функцию $u(x, y)=$ $\operatorname{Re} i z^{n_{0}-1}$. Так как в окрестности бесконечно удаленной точки $\rho_{1}(x)<C|x|^{\varepsilon}$ для любого $\varepsilon>0$, в силу леммы 1 с некоторой постоянной $C_{1}$

$$
\begin{aligned}
\|u(x, y)\|_{L^{\prime}(\rho)} & <\int_{-\infty}^{\infty}\left|(x+i y)^{n_{0}-1}-(x-i y)^{n_{0}-1}\right| \rho(x) d x \\
& <C_{1} y\left(1+\int_{-\infty}^{\infty} \frac{d x}{(1+|x|)^{1+\left\{\alpha_{0}\right\}-\varepsilon}}\right)
\end{aligned}
$$

Взяв $\varepsilon<\left\{\alpha_{0}\right\}$, получим

$$
\lim _{y \rightarrow+0}\left\|\operatorname{Re} i z^{n_{0}-1}\right\|_{L^{1}(\rho)}=0 .
$$

Если $\alpha_{0}$ - целое число, $u(x, y)=\operatorname{Re} i z^{n_{0}-1}$ и имеет место условие $(17)$, то

$$
\|u(x, y)\|_{L^{1}(\rho)}<C y\left(1+\int_{-\infty}^{\infty} \frac{\rho_{1}(x) d x}{1+|x|}\right)
$$

и $\|u(x, y)\|_{L^{1}(\rho)} \rightarrow 0$ при $y \rightarrow+0$.

Рассмотрим случай, когда нарушено условие (17). Тогда функция $u(x, y)=\operatorname{Re} i z^{n_{0}-1}$ в силу леммы 1 при $|x|>2 n_{0}$ у удовлетворяет неравенству $u(x, y)>a y|x|^{n_{0}-2}$ и поэтому не принадлежит классу $L^{1}(\rho)$. Теорема доказана.

Теорема 2. Пусть $\rho(x) \in R$. Тогда задача (2) разрешима для любой функиии $f(x) \in L^{1}(\rho)$. Общее решение можно представить в виде

$$
u(x, y)=u_{0}(x, y)+u_{1}(x, y)
$$

где $u_{0}(x, y)$ - общее решение однородной задачи, $а u_{1}(x, y)$ определяется формулой (16).

ДокАЗАТЕЛЬСТво. Пусть $u(x, y)$ удовлетворяет условию (2). Тогда вьполняется $(13)$ и $u(x, y)$ представима в виде (19). Нам следует установить, что для любой функции $f \in L^{1}(\rho)$ функция $u(x, y)$ из (19) удовлетворяет условию (2). Учитьвая теорему 1 , достаточно установить, что функция $u_{1}(x, y)$ из (16) удовлетворяет условию (2) при любой $f \in L^{1}(\rho)$. Для этого предварительно докажем оценку

$$
\left\|u_{1}(x, y)\right\|_{L^{1}(\rho)}<A\|f\|_{L^{1}(\rho)} \cdot
$$

Заметим, что $u_{1}(x, y)=I_{1}(x, y)+I_{2}(x, y)$, где

$$
\begin{aligned}
& I_{1}(x, y)=\frac{(z+i)^{n_{0}-1}}{4 \pi i} \int_{-\infty}^{\infty} \frac{f(t) d t}{(t+i)^{n_{0}-1}(t-z)}-\frac{(\bar{z}+i)^{n_{0}-1}}{4 \pi i} \int_{-\infty}^{\infty} \frac{f(t) d t}{(t+i)^{n_{0}-1}(t-\bar{z})} \\
& I_{2}(x, y)=\frac{(\bar{z}-i)^{n_{0}-1}}{4 \pi i} \int_{-\infty}^{\infty} \frac{f(t) d t}{(t-i)^{n_{0}-1}(t-z)}-\frac{(z-i)^{n_{0}-1}}{4 \pi i} \int_{-\infty}^{\infty} \frac{f(t) d t}{(t-i)^{n_{0}-1}(t-\bar{z})} .
\end{aligned}
$$


Далее, имеем $I_{1}(x, y)=I_{1}^{(1)}(x, y)+I_{2}^{(2)}(x, y)$, где

$$
\begin{aligned}
& I_{1}^{(1)}(x, y)=\frac{(z+i)^{n_{0}-1}-(\bar{z}+i)^{n_{0}-1}}{4 \pi i} \int_{-\infty}^{\infty} \frac{f(t) d t}{(t+i)^{n_{0}-1}(t-z)} \\
& I_{1}^{(2)}(x, y)=\frac{(\bar{z}+i)^{n_{0}-1}}{2 \pi} \int_{-\infty}^{\infty} \frac{f(t) d t}{(t+i)^{n_{0}-1}(t-x)^{2}+y^{2}} .
\end{aligned}
$$

Учитьвая лемму 1 , получим

$$
\begin{aligned}
\int_{\infty}^{\infty} & \left|I_{1}^{(1)}(x, y)\right| \rho(x) d x \\
& =\sup _{g \in B_{0}}\left|\int_{\infty}^{\infty}\left((z+i)^{n_{0}-1}-(\bar{z}+i)^{n_{0}-1}\right) \int_{\infty}^{\infty} \frac{f(t) d t}{(t+i)^{n_{0}-1}(t-x-i y)} g(x) d x\right| \\
& <C \int_{\infty}^{\infty}|f(t)| \rho(t) \frac{|t+i|^{\{\alpha\}}}{\rho_{1}(t)} \int_{\infty}^{\infty} \frac{y\left(y^{n_{0}-2}+|x|^{n_{0}-2}\right)|x|^{1-\{\alpha\}} \rho_{1}(x)}{|x+i|^{n_{0}}|t-x-i y|} d x d t \\
& <C_{1} \int_{\infty}^{\infty}|f(t)| \rho(t) \frac{|t+i|^{\left\{\alpha_{0}\right\}}}{\rho_{1}(t)} \int_{\infty}^{\infty} \frac{y \rho_{1}(x)}{|x+i|^{1+\left\{\alpha_{0}\right\}}|t-x-i y|} d x d t
\end{aligned}
$$

где $B_{0}=\left\{g:\|g\|_{L^{\infty}\left(\rho^{-1}\right)} \leqslant 1\right\}$. Отсюда, учитывая $(12)$, получаем оценку

$$
\left\|I_{1}^{(1)}(x, y)\right\|_{L^{1}(\rho)}<C\|f\|_{L^{1}(\rho)} .
$$

Повторяя те же выкладки и применяя (11), получим

$$
\left\|I_{1}^{(2)}(x, y)\right\|_{L^{1}(\rho)}<C\|f\|_{L^{1}(\rho)} .
$$

Следовательно,

$$
\left\|I_{1}(x, y)\right\|_{L^{1}(\rho)}<C\|f\|_{L^{1}(\rho)} .
$$

Аналогично доказьвается, что

$$
\left\|I_{2}(x, y)\right\|_{L^{1}(\rho)}<C\|f\|_{L^{1}(\rho)} .
$$

Тем самым, оценка (20) доказана. Докажем, что из (20) следует, что функция (16) удовлетворяет условию (2). Положим

$$
f_{n}(x)= \begin{cases}0, & |x|>n, \\ f(x), & |x|<n .\end{cases}
$$

Получим последовательность функций $f_{n}(x)$ такую, что

$$
\left\|f_{n}(x)-f(x)\right\|_{L^{1}(\rho)} \rightarrow 0 .
$$

Обозначив функцию $(16)$ при $f(x)=f_{n}(x)$ через $u_{n}(x, y)$, будем иметь (см. [8])

$$
\lim _{y \rightarrow+0}\left\|u_{n}(x, y)-f_{n}(x)\right\|_{L^{1}(\rho)}=0 .
$$

Далее, используя оценку (20), получим

$$
\begin{aligned}
\|u(x, y)-f(x)\|_{L^{1}(\rho)}< & \left\|u(x, y)-u_{n}(x, y)\right\|_{L^{1}(\rho)}+\left\|u_{n}(x, y)-f_{n}(x)\right\|_{L^{1}(\rho)} \\
& +\left\|f_{n}(x)-f(x)\right\|_{L^{1}(\rho)} \\
< & C\left\|f_{n}(x)-f(x)\right\|_{L^{1}(\rho)}+\left\|u_{n}(x, y)-f_{n}(x)\right\|_{L^{1}(\rho)} .
\end{aligned}
$$

Из (21) и (22) теперь следует, что $u(x, y)$ удовлетворяет условию (2). Теорема доказана. 


\section{СПИСОК ЦИТИРОВАННОЙ ЛИТЕРАТУРЫ}

[1] Хведелидзе Б. В. О разрьвной задаче Римана-Привалова для нескольких функций // Сообщение АН Груз. ССР. 1956. Т. 17. №10. С. 865-872.

[2] Rosenblum M. Summability of Fourier series in $L_{p}(d \mu) / /$ Trans. Amer. Math. Soc. 1962. V. 165. P. 326-342.

[3] Товмасян Н. Е. О существовании и единственности решения задачи Дирихле для уравнения Лапласа в классе функций, имеющих особенности на границе // Сиб. матем. ж. 1961. Т. 2. № 2. C. $25-57$.

[4] Гохберг И. Ц., Крупник Н. Я. Введение в теорию одномерных сингулярных интегральных операторов. Кишинёв, 1973.

[5] Hunt R.A., Muckenhoupt B., Wheeden R. L. Weighted norm inequalities for the conjugate functions and Hilbert transform // Trans. Amer. Math. Soc. 1973. V. 176. P. 227-251.

[6] Хведелидзе Б. В. Методы интегралов типа Коши в разрывных граничных задачах теории голоморфных функций одной комплексной переменной // Современные проблемы математики. T. 7. M., 1975.

[7] Прёсдорф З. Некоторые классы сингулярных уравнений. М.: Мир, 1979.

[8] Гарнетт Дж. Ограниченные аналитические функции. М.: Мир, 1984.

[9] Kazarian K.S. Weighted norm inequalities for some classes of singular integrals // Studia Math. 1987. V. 86. P. 97-130.

[10] Айрапетян Г. М. Задача Дирихле в пространствах с весом // Изв. НАН Армении. Матем. 2001. T. 36. № 3. C. 12-35.

[11] Айрапетян Г. М. О задаче Дирихле в полуплоскости в пространствах с весом // Изв. НАН Армении. Матем. 2001. Т. 36. №6. С. 7-15.

[12] Айрапетян Г. М. О задаче Гильберта в смысле $L^{p}$-сходимости, $p>1 / /$ Докл. РАН. 1993. T. 328. № 4. С. 421-423.

[13] Сенета Е. Правильно меняюшиеся функции. М.: Наука, 1985.

Государственный инженерный университет Армении

Поступило

E-mail : hhairapet@seua.am

24.01.2003 